\title{
STAGEWISE MULTIDIMENSIONAL VISUAL DISCRIMINATION BY PIGEONS
}

\author{
Olga V. Vyazovska ${ }^{1}$, Victor M. Navarro ${ }^{2}$, and Edward A. Wasserman ${ }^{2}$ \\ ${ }^{\mathrm{l} V}$. N. KARAZIN KHARKIV NATIONAL UNIVERSITY, UKRAINE \\ ${ }^{2}$ THE UNIVERSITY OF IOWA, USA
}

\begin{abstract}
We trained six pigeons in a stagewise Multiple Necessary Cues (MNC) go/no-go task to document the dynamics of discrimination learning involving increasingly complex visual stimuli. The compound stimuli were composed from four dimensions, each of which could assume either of two extreme values or their intermediate value: Shape, Size, Line Orientation, and Brightness. Starting with a stimulus composed entirely from intermediate values, we replaced those values with each of the two extreme dimensional values in four successive stages, thereby increasing the stimulus set from 2 in Stage 1 to 16 in Stage 4 . In each stage, only one combination of values signaled food $\left(\mathrm{S}^{+}\right)$, whereas the remaining combinations did not $\left(\mathrm{S}^{-} \mathrm{s}\right)$. We calculated the rate of pecking during the first $15 \mathrm{~s}$ of each stimulus presentation and, in any given stage, training continued until the rate of responding to all of the $\mathrm{S}^{-} \mathrm{s}$ was less than $20 \%$ of the rate of responding to the $\mathrm{S}^{+}$. All pigeons successfully acquired the final discrimination, suggesting that they attended to all of the dimensions relevant for the discrimination. We also replicated the key results of prior MNC studies: (1) the number of extreme dimensional values in each stage was positively related to the amount of training required for pigeons to acquire the discrimination; (2) attentional tradeoffs were most often observed when three or four dimensions were being trained; and (3) throughout training, the number of dimensional values in the $\mathrm{S}^{-} \mathrm{s}$ that differed from the $\mathrm{S}^{+}$was positively related to their discriminability from $\mathrm{S}^{+}$.

Key words: visual discrimination learning, attentional tradeoff, dimensional stimulus control, configural stimulus control, pigeon
\end{abstract}

The issue of multidimensional stimulus control remains a central problem for behavioral science. Several approaches have been deployed to study the control that each of the dimensions of a compound stimulus can exert over behavior (Chatlosh \& Wasserman, 1993; Riley \& Roitblat, 1978; Soto \& Wasserman, 2010; Sutherland \& Mackintosh, 1971; Thomas, 1970; Zentall, 2012). One of those approaches is represented by the Multiple Necessary Cues (MNC) discrimination task, a task that poses strong challenges to the learner. Consider the case of stimuli composed of features chosen from pairs of values lying along four orthogonal dimensions: A1 and $\mathrm{A} 2, \mathrm{~B} 1$ and $\mathrm{B} 2, \mathrm{C} 1$ and $\mathrm{C} 2$, and $\mathrm{D} 1$ and D2. All possible combinations yield 16 unique compound stimuli. The learner must respond

We thank D. V. Pavlenko in the Department of Physical and Biomedical Electronics and Complex Information Technologies, Radiophysics faculty, V. N. Karazin Kharkiv National University, and D. A. Shelkovenkov, Ph.D., Test Engineer at Melexis-Ukraine, for their technical support.

Correspondence concerning this article should be addressed to Edward A. Wasserman, Department of Psychological and Brain Sciences, E11 Seashore Hall, The University of Iowa, Iowa City, IA 52242. Email: ed-wasserman@uiowa.edu

doi: $10.1002 /$ jeab.217 in one way to the positive compound stimulus A1B1C1D1 $\left(\mathrm{S}^{+}\right)$and in a second, distinctive way to the 15 negative compound stimuli $\left(\mathrm{S}^{-} \mathrm{s}\right)$; those two response options can be afforded in either simultaneous choice (Teng, Vyazovska, \& Wasserman, 2015) or successive go/no-go problems (Vyazovska, Teng, \& Wasserman, 2014). In either case, a learner's attention would appear to be demanded to each of the four dimensions of the stimuli and possibly to the unique configural cues arising from the 16 stimulus combinations.

If this discrimination task is arranged so that all four stimulus dimensions are relevant for the discrimination, then pigeons readily solve it (Vyazovska et al., 2014; Teng et al., 2015). In doing so, the pigeons show three patterns of performance during acquisition. First, the speed of discrimination learning is positively related to the number of dimensional disparities between the $\mathrm{S}^{+}$and $\mathrm{S}^{-}$compounds: 4 disparities $>3$ disparities $>2$ disparities $>$ 1 disparity, suggesting that stimulus generalization between the $\mathrm{S}^{+}$and $\mathrm{S}^{-}$compounds plays a key part in the discrimination learning process (Vyazovska et al., 2014; Teng et al., 2015). Second, separately scoring discriminative responding to each stimulus dimension can yield differences in the speed of learning that 
depend on the specific stimulus values that are chosen along each of the different dimensions (Gottselig, Wasserman, \& Young, 2001). Finally, those individual dimensional discrimination indexes often exhibit what appear to be robust attentional tradeoffs: namely, rises in discrimination along one dimension closely accompanied by falls in discrimination along one or two other dimensions (Vyazovska et al., 2014).

Rather than having all four of the stimulus dimensions being relevant from the outset of training, the MNC discrimination can be sequentially arranged across stages, by having one, then two, then three, and finally all four dimensions become relevant to the task (Gottselig et al., 2001). In Stage 1, the $\mathrm{S}^{+}$takes the form of A1BXCXDX (with $\mathrm{X}$ denoting either of the two values for each dimension), and any other combination of values is an $S^{-}$ (i.e. A2BXCXDX). The discrimination is then made progressively more complex. In Stage 2 , in which a second dimension becomes relevant, the $\mathrm{S}^{+}$now takes the form of A1B1CXDX. In Stage 3, when a third dimension becomes relevant, the $\mathrm{S}^{+}$takes the form of A1B1C1DX. Finally, in Stage 4 the discrimination task turns into the same as the standard MNC task: The fourth dimension becoming relevant makes the $\mathrm{S}^{+}$take the form of A1B1C1D1, with every other combination being an $\mathrm{S}^{-}$.

That stagewise training may afford analytical and statistical advantages because learners must attend to the programmed dimensions in a determinate order. However, the sequential training procedure of Gottselig and collaborators (2001) entailed an interpretive complication: Both of the newly relevant dimensional values had previously been presented and had been irrelevant to the arranged contingencies of reinforcement. Therefore, learned irrelevance-the reduction of attention to stimuli that do not predict changes in the likelihood of reinforcement-might have arisen and participated in the results (Mackintosh, 1973; Mackintosh, 1975; Treisman, 1964).

In the present project, we also arranged a stagewise MNC task, but we minimized the contribution of learned irrelevance by arranging single, intermediate values along the irrelevant dimensions. When those dimensions became relevant in subsequent stages of training, their intermediate values were replaced by the two dimensional values necessary for the discrimination (Fig. 1). Under these conditions, the results should not be complicated by decrements in attention, thereby providing an unvarnished look at the dynamics of discrimination performance under the stagewise MNC task.

\section{Method}

\section{Subjects}

Six experimentally naïve adult pigeons (Columba livia) of mixed sex were studied; they were kept in individual $50 \times 50 \times 50 \mathrm{~cm}$ cages under a 12:12 hr light/dark cycle (lights on at 08:00). Throughout the experiment, the pigeons were maintained at $85 \%$ of their freefeeding weights, with water freely available in the home cages.

\section{Apparatus}

The pigeons were trained in a standard operant conditioning chamber $(35 \times 35 \times 35 \mathrm{~cm})$. The front wall of the chamber contained a transparent rectangular frame $(9.50 \mathrm{~cm}$ high $\times$ $17.50 \mathrm{~cm}$ wide) made of Plexiglas. The bottom edge of the frame was $15.50 \mathrm{~cm}$ above the floor; the left and right edges were equidistant from the sidewalls. Immediately behind the frame was a resistive touchscreen; pecks to the touchscreen were limited to the opening in the rectangular frame and were processed by a controller board outside the chamber. A CRT display was located $2 \mathrm{~mm}$ behind the touchscreen. A food delivery cup was placed in the floor directly beneath the touchscreen opening; mixed grain reinforcers could be dispensed into this cup via a rotary delivery mechanism. During experimental sessions, the chamber was constantly illuminated by a $1.5 \mathrm{~W}$ incandescent lamp located near the top of the rear wall. A PC running MatLab 2010b ${ }^{\circledR}$ software controlled discriminative stimulus presentation, the delivery of food, and the recording of touchscreen responses.

\section{Stimuli}

We prepared one compound stimulus with intermediate values of each dimension (Fig. 1, top row), shaped as a square/circle morph, with vertical and horizontal dimensions of $4.7 \mathrm{~cm}$, a diagonally oriented line $\left(45^{\circ}\right)$, and medium brightness (RGB 135, 135, 135), plus 72 other stimuli with one, two, three, or all 


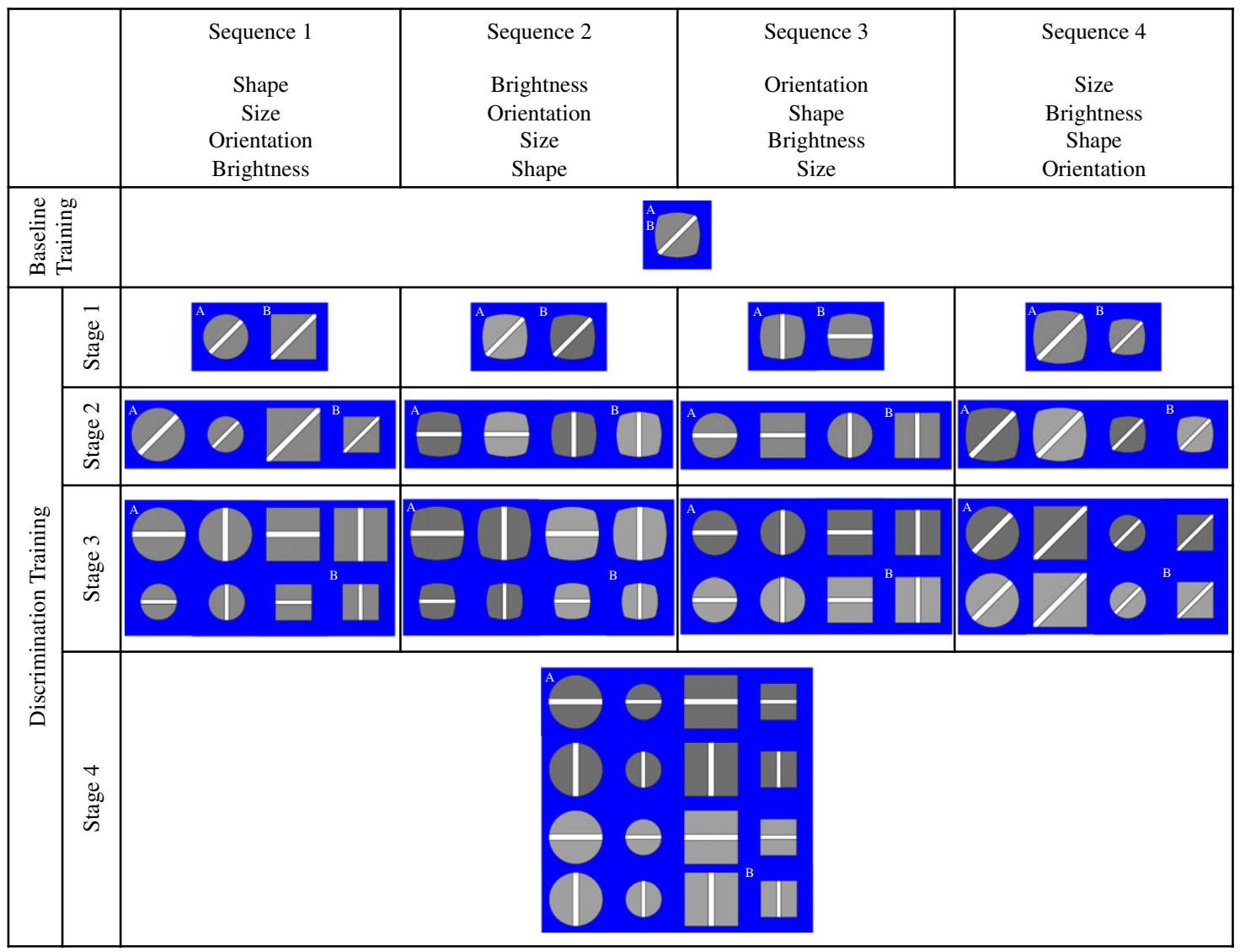

Fig. 1. Stimulus sets presented in each session across stages for each of the four pigeon subgroups. During baseline training, a figure composed of intermediate values was shown. Discrimination training consisted of four stages. In each stage, one of the intermediate dimensional values in the previous stage was replaced by extreme (nonintermediate) values. Different pigeons had different training sequences (columns) and different endpoint $\mathrm{S}+\mathrm{s}$ (not shown), but ultimately reached the same 16 stimulus set. Stimuli marked with A or B served as S + s, depending on the pigeon's training condition. The remaining stimuli served as $\mathrm{S}^{-} \mathrm{s}$, regardless of the pigeon's training condition. See the online version of this article for a colored version of this figure.

four of these intermediate dimensional values replaced by one of two extreme, nonintermediate values: Shape (Circle or Square), Size (Large or Small), Orientation (Horizontal or Vertical line), and Brightness (Dark or Light). The full set of stimuli is shown in Figure 1. These dimensions were chosen because of their proven effectiveness (Castro \& Wasserman, 2013; Gottselig et al., 2001; Vyazovska et al., 2014) and perceptual separability (Soto \& Wasserman, 2010).

The diameter of the large circle and the side of the large square was $5.6 \mathrm{~cm}$; the diameter of the small circle and the side of the small square was $3.8 \mathrm{~cm}$. The RGB values of the dark brightness were $110,110,110$; the RGB values of the light brightness were 160, 160, 160 .

All of the discriminative stimuli were presented in the center of the touchscreen frame on a blue background (RGB $0,0,255$ ), which filled the entire display. The effective pecking area containing each discriminative stimulus (Large or Small) was $6.4 \times 6.4 \mathrm{~cm}$ in order to equate the opportunity to record pecks from stimuli of different sizes.

\section{Procedure}

Shaping. Because the pigeons were experimentally naïve, they first had to be trained to 
eat from the food cup and to peck the touchscreen. We thus conducted manual shaping with the same discriminative stimulus as would later be used in Baseline Training.

Baseline training. During baseline training, pigeons were given daily sessions composed of 160 presentations of the stimulus involving intermediate values of each dimension, with a random intertrial interval (ITI) averaging $8 \mathrm{~s}$ and a range of 6 to $10 \mathrm{~s}$. The first peck within the effective pecking area after $15 \mathrm{~s}$ of stimulus presentation was reinforced with food. Each pigeon received baseline training until its mean response rate to the stimulus was stable (i.e. daily session response rates differed by no more than 0.2 pecks per $\mathrm{s}$ for at least three sessions).

Discrimination training. Three pigeons had SSVL (shape $=$ Square, size $=$ Small, line orientation $=$ Vertical, brightness $=$ Light) as their endpoint $\mathrm{S}^{+}$; the other three pigeons had CLHD (shape $=$ Circle, size $=$ Large, line orientation $=$ Horizontal, brightness $=$ Dark) as their endpoint $\mathrm{S}^{+}$. We introduced the extreme (nonintermediate) values of the four different dimensions using four different sequences (involving the columns illustrated in Fig. 1). The identifiers of the pigeons denote the sequence ( 1 to 4$)$ in which the extreme dimensional values were introduced and their endpoint $\mathrm{S}^{+}$(A or B for SSVL and CLHD, respectively). For example, Pigeon $1 \mathrm{~A}$ was trained using Sequence 1 (Shape, Size, Orientation, and Brightness) and its endpoint $\mathrm{S}^{+}$ was stimulus SSVL. Two pigeons were trained using Sequence 1 (1A and $1 \mathrm{~B}$ ), one using Sequence 2 (2B), one using Sequence 3 (3A), and two using Sequence 4 ( $4 \mathrm{~A}$ and $4 \mathrm{~B}$ ).

Pigeons were given four stages of discrimination training, with increasingly numerous stimulus sets. In Stage 1, the stimulus set consisted of all combinations between one pair of extreme (nonintermediate) dimensional values and the remaining three intermediate values, yielding a total of two stimuli. In Stage 2, a new pair of extreme values replaced the intermediate value of another dimension; therefore, the stimulus set consisted of all combinations between two pairs of extreme dimensional values and the remaining two intermediate values, yielding a total of four stimuli. In Stage 3, a third pair of extreme values replaced the intermediate value of another dimension, yielding a total of eight stimuli. Finally, in Stage 4 , the stimuli had no intermediate dimensional values, and the stimulus set consisted of all possible combinations between the four pairs of extreme values, expanding the set size to 16 stimuli.

To make this process even clearer, take pigeon $1 \mathrm{~B}$ as an example. This pigeon was trained using Sequence 1, which had CLHD as its endpoint $\mathrm{S}^{+}$. In Stage 1, the discriminative stimuli were a circle and a square-each of intermediate brightness, orientation, and size-and the pigeon received food only for pecking the circle $(\mathrm{C})$. In Stage 2, the discriminative stimuli were two circles (one small and one large) and two squares (one small and one large) of intermediate brightness and line orientation, and the pigeon received food only for pecking the large circle (CL). In Stage 3 , the discriminative stimuli were now four circles and four squares each differing in size (large or small) and line orientation (horizontal or vertical), but with intermediate brightness. During this stage, reinforcers only followed pecks at the large circle with a horizontal line (CLH). Finally, when the brightness dimension was made relevant in Stage 4 , the discriminative stimuli were eight different circles and eight different squares, and the pigeon received food for pecking the dark, large circle with a horizontal line (CLHD). It is important to note that an extreme $\mathrm{S}^{+}$value from a prior stage (e.g., C) continued to be a component of the $\mathrm{S}^{+}$stimulus in subsequent stages (e.g., CL), but that responding to it was only reinforced if it appeared with the other positive dimensional values.

In discrimination training, 10 blocks of 16 trials each were presented in daily training sessions, for a total of 160 trials, with a random ITI of 6 to $10 \mathrm{~s}$. The order of stimulus presentation was randomized within blocks. On $\mathrm{S}^{+}$trials, the discriminative stimulus remained on the screen for at least $15 \mathrm{~s}$; the first peck after $15 \mathrm{~s}$ turned off the stimulus and delivered 1 to $2 \mathrm{~s}$ of food in baseline training and the first training stage, 2 to $3 \mathrm{~s}$ of food in the second training stage, 2 to $5 \mathrm{~s}$ of food in the third training stage, and $5 \mathrm{~s}$ of food in the fourth training stage. These durations of food delivery maintained the pigeons' weights at the $85 \%$ level, because the number of trials per session that involved food reinforcers progressively fell across stages. On $\mathrm{S}^{-}$ 
trials, the discriminative stimulus remained on the screen for exactly $15 \mathrm{~s}$, and it was followed immediately by the ITI. In any stage, once the pigeon's response rate to all of the $\mathrm{S}^{-} \mathrm{s}$ in a single daily session was $20 \%$ or less than its rate to the $\mathrm{S}^{+}$, the pigeon was moved to the next discrimination stage until all four stages were completed. In order to equate the opportunity for responding on $\mathrm{S}^{+}$and $\mathrm{S}^{-}$ trials, only those pecks that occurred during the first $15 \mathrm{~s}$ of stimulus presentation were used in the data analyses.

\section{Results}

Discrimination learning. All six pigeons learned the stagewise four-dimensional discrimination task, taking them a mean of 37.7 $(\mathrm{SD}=18.2)$ sessions to do so. Considerable individual differences in the speed of learning were observed. The fastest pigeon (3A) completed all four training stages in only 16 sessions, whereas the slowest pigeon (2B) required 66 sessions. Table 1 shows the mean rate of responding for each pigeon to all 16 discriminative stimuli on the final session of Stage 4 of discrimination training. Clearly, the stagewise MNC task was learnable by the pigeons.
The pigeons generally took longer to complete the two later, demonstrably more difficult, stages of training. On average, the first and second stages lasted 2.5 $(\mathrm{SD}=1.0)$ and $3.0 \quad(\mathrm{SD}=0.9)$ sessions, respectively, whereas the third and fourth stages lasted 8.5 $(\mathrm{SD}=3.8) \quad$ and $\quad 23.7 \quad(\mathrm{SD}=18.0) \quad$ sessions, respectively.

Dimensional stimulus control. We explored whether the four dimensions differentially controlled the pigeons' responding by examining each pigeon's responses to each of the four dimensions across all of its training sessions. A discrimination ratio (DR) for each dimension was calculated using the formula:

$$
\mathrm{DR}_{\mathrm{D}}=\frac{\overline{\mathrm{RR}}_{\mathrm{d}+}}{\left(\overline{\mathrm{RR}}_{\mathrm{d}-}+\overline{\mathrm{RR}}_{\mathrm{d}+}\right)}
$$

where $D_{D}$ is the discrimination ratio of dimension $\mathrm{D}, \overline{\mathrm{RR}}_{\mathrm{d}+}$ is the mean response rate to all stimuli involving the dimension's $\mathrm{S}^{+}$ value, and $\overline{R R}_{d}$ - is the mean response rate to all stimuli involving the dimension's $\mathrm{S}^{-}$value. A DR of 1.00 indicates perfect discrimination between the dimension's $\mathrm{S}^{+}$and $\mathrm{S}^{-}$values, whereas a DR of .50 indicates no discrimination.

Table 1

Mean Response Rate on Final Session of Discrimination Training.

\begin{tabular}{|c|c|c|c|c|c|c|}
\hline \multirow[b]{4}{*}{ Stimulus } & \multicolumn{6}{|c|}{ Response Rate (Pecks/s) } \\
\hline & \multicolumn{6}{|c|}{ Pigeon (Sessions of Training) } \\
\hline & \multicolumn{3}{|c|}{ SSVL+ Condition } & \multicolumn{3}{|c|}{ CLHD+ Condition } \\
\hline & 1A (34) & $3 \mathrm{~A}(16)$ & $4 \mathrm{~A}(51)$ & $1 \mathrm{~B}(24)$ & 2B (66) & $4 \mathrm{~B}(35)$ \\
\hline CLHD & 0.03 & 0.00 & 0.09 & 0.21 & 3.86 & 0.67 \\
\hline CLHL & 0.04 & 0.00 & 0.09 & 0.01 & 0.06 & 0.00 \\
\hline CLVD & 0.01 & 0.00 & 0.08 & 0.00 & 0.06 & 0.00 \\
\hline CLVL & 0.04 & 0.01 & 0.17 & 0.00 & 0.04 & 0.00 \\
\hline CSHD & 0.01 & 0.00 & 0.06 & 0.00 & 0.72 & 0.07 \\
\hline CSHL & 0.01 & 0.01 & 0.11 & 0.00 & 0.03 & 0.00 \\
\hline CSVD & 0.00 & 0.00 & 0.09 & 0.00 & 0.04 & 0.00 \\
\hline CSVL & 0.03 & 0.00 & 0.26 & 0.00 & 0.04 & 0.00 \\
\hline SLHD & 0.01 & 0.00 & 0.06 & 0.00 & 0.67 & 0.07 \\
\hline SLHL & 0.02 & 0.00 & 0.20 & 0.00 & 0.04 & 0.00 \\
\hline SLVD & 0.04 & 0.01 & 0.08 & 0.00 & 0.01 & 0.00 \\
\hline SLVL & 0.01 & 0.07 & 0.43 & 0.00 & 0.02 & 0.00 \\
\hline SSHD & 0.05 & 0.00 & 0.05 & 0.01 & 0.45 & 0.01 \\
\hline SSHL & 0.22 & 0.01 & 0.45 & 0.00 & 0.03 & 0.00 \\
\hline SSVD & 0.08 & 0.01 & 0.09 & 0.00 & 0.03 & 0.00 \\
\hline SSVL & 1.35 & 0.94 & 2.87 & 0.00 & 0.05 & 0.00 \\
\hline
\end{tabular}

Note: Response rates to the $\mathrm{S}^{+}$compound are in bold. 
Table 2

Mean Discrimination Ratios for Each Different Dimension.

\begin{tabular}{|c|c|c|c|c|c|c|c|}
\hline \multirow[b]{4}{*}{ Dimension } & \multicolumn{7}{|c|}{ Discrimination Ratio: $R R$ to $S^{+} /\left(R R\right.$ to $S^{-} S+R R$ to $\left.S^{+}\right)$} \\
\hline & \multicolumn{7}{|c|}{ Pigeon (Sessions of Training) } \\
\hline & \multicolumn{3}{|c|}{ SSVL+ Condition } & \multicolumn{3}{|c|}{ CLHD+ Condition } & \multirow[b]{2}{*}{ Mean (37.7) } \\
\hline & $1 \mathrm{~A}(34)$ & $3 \mathrm{~A}(16)$ & $4 \mathrm{~A}(51)$ & 1B (24) & 2B (66) & $4 \mathrm{~B}(35)$ & \\
\hline Shape & .88 & .87 & .67 & .87 & .58 & .65 & .75 \\
\hline Size & .85 & .68 & .73 & .79 & .81 & .87 & .79 \\
\hline Orientation & .62 & .91 & .79 & .84 & .97 & .98 & .85 \\
\hline Brightness & .90 & .93 & .95 & .80 & .97 & .99 & .92 \\
\hline
\end{tabular}

Note $:$ RR = Response Rate.

Table 2 shows the DRs for each dimension, averaged across the totality of training. Overall, the pigeons appeared to have had the greatest difficulty discriminating the shape dimension, followed by the size, orientation, and brightness dimensions; three out of the six pigeons $(4 \mathrm{~A}, 2 \mathrm{~B}$, and $4 \mathrm{~B})$ exhibited this pattern of performance. However, a one-way Analysis of Variance (ANOVA) of the pigeons' overall dimensional DRs found that dimension did not reliably affect the pigeons' discrimination $(p>.10)$. Although there were reliable differences among the dimensions for individual pigeons, they were somewhat idiosyncratic (see Table S1 in the supplemental materials). These results suggest that it is unlikely the four dimensions differed in inherent salience or discriminability (cf. Vyazovska et al., 2014).

Control by number of dimensional disparities. To see whether the pigeons' discriminative responding depended on how discrepant each $\mathrm{S}^{-}$was from the $\mathrm{S}^{+}$, we categorized the $\mathrm{S}^{-} \mathrm{S}$ according to the number dimensional values each $\mathrm{S}^{-}$differed from the $\mathrm{S}^{+}$: by one dimensional disparity (DD1), by two dimensional disparities (DD2), by three dimensional disparities (DD3), and by four dimensional disparities (DD4). (See Table 3 for an example depicting how the different compound stimuli were categorized across stages for Pigeon 1B.) After categorizing the compounds, we calculated a DR for each of these four $\mathrm{S}^{-}$categories with the formula:

$$
\mathrm{DR}_{\mathrm{DDn}}=\frac{\mathrm{RR}_{\mathrm{S}^{+}}}{\left(\overline{\mathrm{RR}}_{\mathrm{DDn}}+\mathrm{RR}_{\mathrm{S}^{+}}\right)}
$$

Table 3

Categorization of $\mathrm{S}^{-}$Compounds for Pigeon 1B, Based on their Dimensional Disparities (DD) with the $\mathrm{S}^{+}$ Compound.

\begin{tabular}{lccc}
\hline Stage & S $^{+}$ & S- Compounds & DD \\
\hline \hline 1 & C & S & 1 \\
2 & CL & CS, SL & 1 \\
3 & & SS & 2 \\
& CLH & CLV, CSH, SLH & 1 \\
& & CSV, SSH, SLV & 2 \\
4 & CLHD & CLHV & 3 \\
& & CLVL, CSHD, CSHD, SLHD & 1 \\
& & CSLVD, SLHL, SLV , SSHD & 2 \\
& & CSSHL, SSVD & 3 \\
& & SSVL & 4 \\
\hline
\end{tabular}

Note: The letters in each compound denote its dimensional values: shape $(\mathrm{C}=$ circle, $\mathrm{S}=$ square $)$, size $(\mathrm{L}=$ large, $\mathrm{S}=$ small), orientation $(\mathrm{H}=$ horizontal, $\mathrm{V}=$ vertical $)$, and brightness $(\mathrm{D}=$ dark, $\mathrm{L}=$ light $)$, respectively.

where $\mathrm{DR}_{\mathrm{DDn}}$ is the discrimination ratio for $\mathrm{S}^{-}$ compounds with $\mathrm{n}$ dimensional disparities, $\mathrm{RR}_{\mathrm{S}^{+}}$is the response rate to the $\mathrm{S}^{+}$, and $\overline{\mathrm{RR}}_{\mathrm{DDn}}$ is the mean response rate to all of the $\mathrm{S}^{-} \mathrm{s}$ within the same DD category.

Figure 2 shows the DRs for all four DD categories in each of the training sessions. The pigeons readily discriminated DD4 and DD3 stimuli in the two later stages, they but had more trouble discriminating DD1 and DD2 stimuli, which more closely resembled the $\mathrm{S}^{+}$. To assess these differences, and because stimuli from different DDs were not equally available throughout training-for example, DD1 stimuli were available across Stages 1 to 4, whereas DD4 stimuli were available only in Stage 4 -we took two different approaches to analyze these data. 

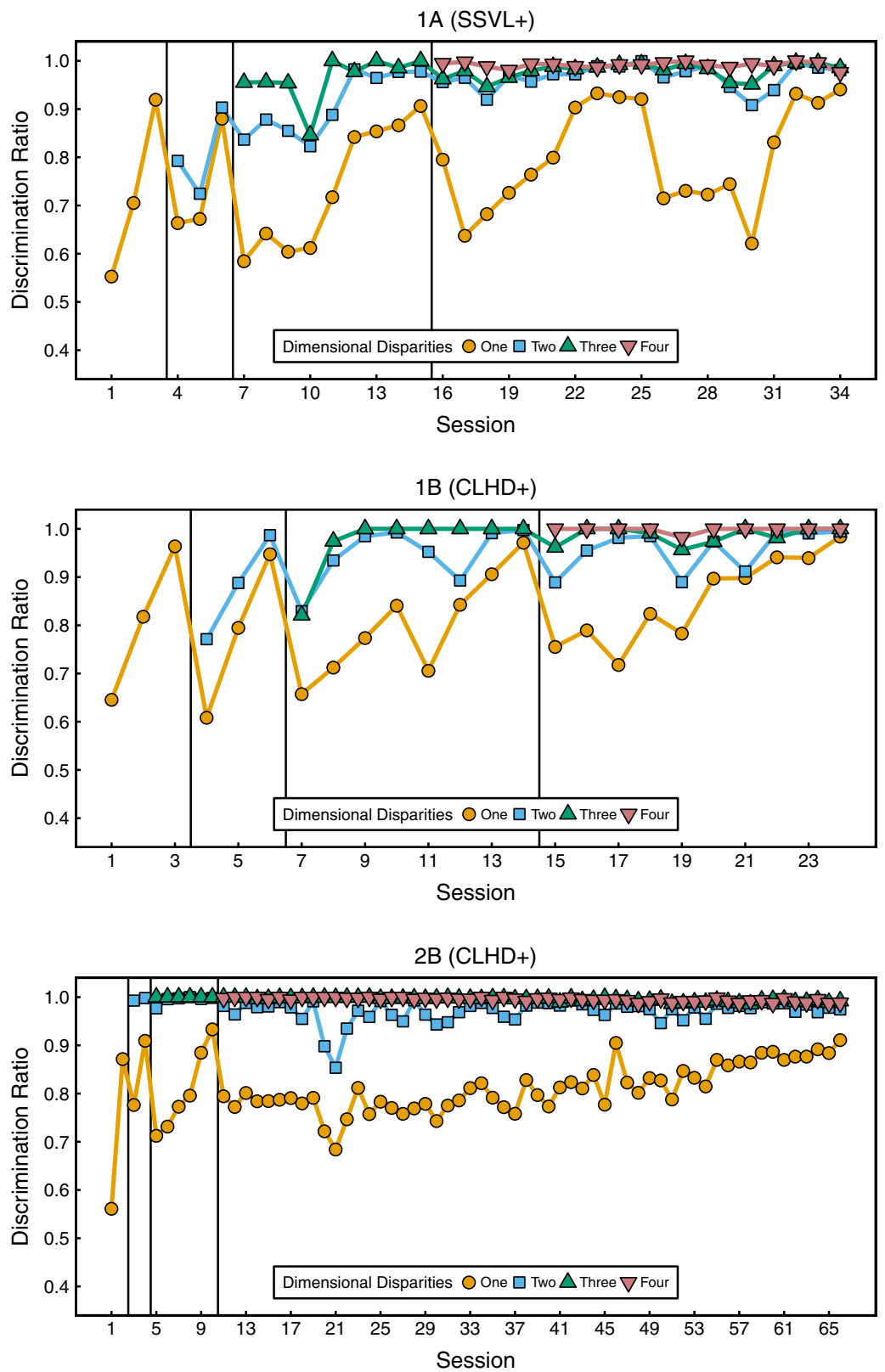

Fig. 2. Discrimination Ratios (DRs) for one, two, three, and four dimensional disparities throughout the entirety of discrimination training for each pigeon. The vertical lines mark the addition of a new dimension. See the online version of this article for a colored version of this figure.

First, we analyzed whether there was an overall effect of the number of DDs in discrimination training. Table 4 shows the average DRs for all DD categories collapsed across training stages. An ANOVA of these DRs revealed a statistically significant effect of the number of dimensional disparities on discrimination performance, $F(3,15)=177.13$, $p<.001, \eta^{2}=0.973$. That effect was further explored by performing multiple pairwise $t$-tests among the DDs, with a Bonferroni correction for multiple comparisons. These comparisons disclosed that discrimination performance with DD1 stimuli $($ mean $=.80)$ 

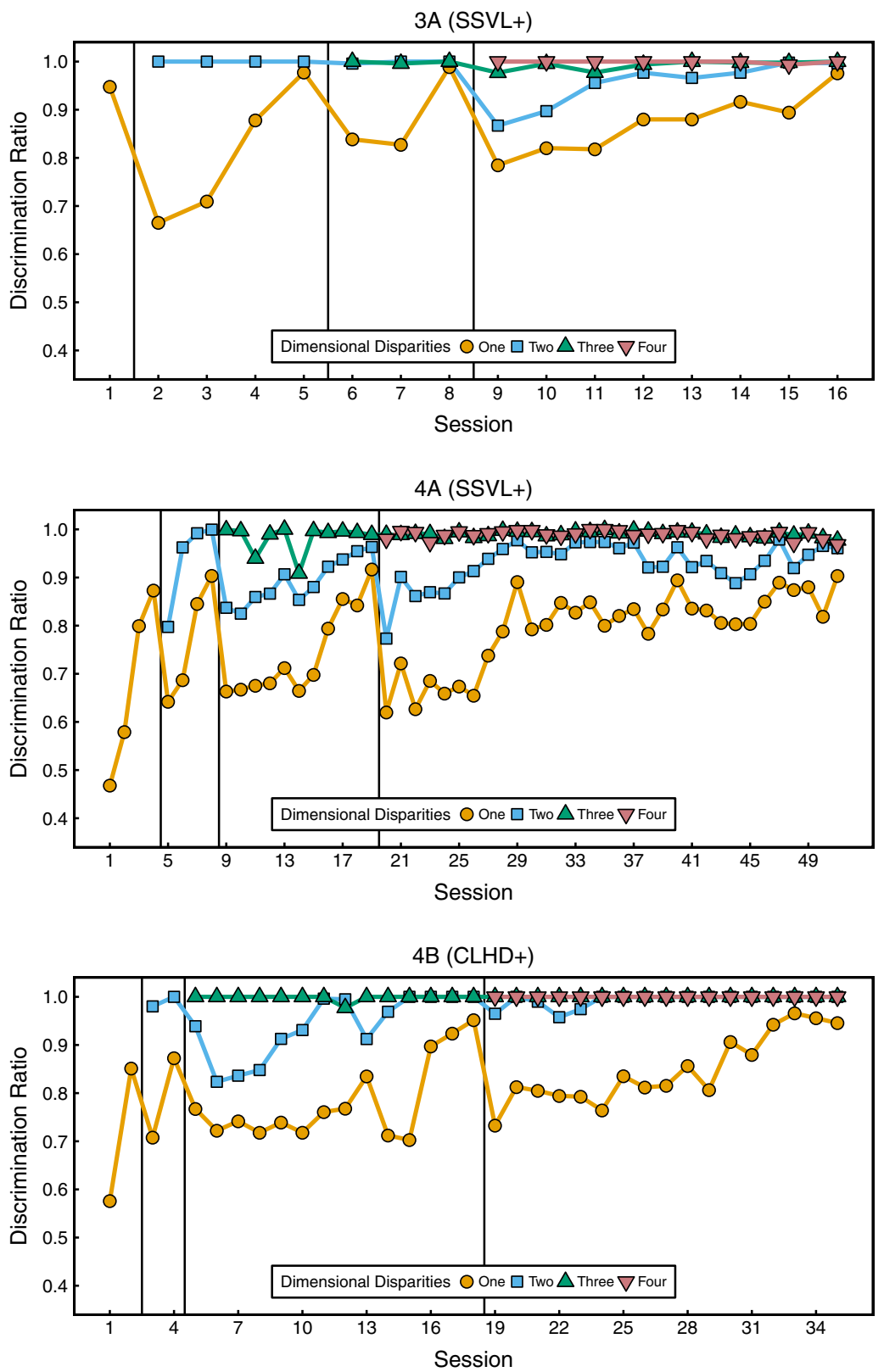

Fig. 2. Continued

was reliably poorer than discrimination performance with DD2 $($ mean $=.95)$, DD3 $($ mean $=$ $.99)$, or DD4 stimuli (mean $=.99$; all $p$ s $<.01)$, that discrimination performance with DD2 stimuli was reliably poorer than discrimination performance with DD3 or DD4 stimuli (both $p$ s $<.05)$, but that discrimination performance was not reliably different between DD3 and DD4 stimuli $(p>.10)$.
Second, we assessed the differences in discrimination performance among the different DD categories in Stages 2 to 4 (Table 5). Discrimination performance was reliably different among DD categories in every stage, $F(1, \quad 5)=46.23, \quad p<.01, \quad \eta^{2}=0.902 ; \quad F$ $(2,10)=95.66, p<.001, \eta^{2}=0.950 ;$ and $F$ $(3,15)=131.2, p<.001, \eta^{2}=0.963$, for Stages 2, 3, and 4, respectively. Planned comparisons 
Table 4

Mean Discrimination Ratios Across All Sessions of Discrimination Training for Different Numbers of Dimensional Disparities (DDs).

\begin{tabular}{lcccc}
\hline & \multicolumn{3}{c}{$\begin{array}{c}\text { Dimensional Disparities (DD) Discrimination } \\
\text { Ratio: }\end{array}$} \\
\cline { 2 - 5 } & \multicolumn{4}{c}{ RR to S $^{+} /\left(\right.$RR to S $^{-}$s + RR to S $\left.{ }^{+}\right)$} \\
\cline { 2 - 5 } & \multicolumn{4}{c}{ Dimensional Disparities } \\
\cline { 2 - 5 } Pigeon & 1 & 2 & 3 & 4 \\
\hline \hline 1A & .77 & .93 & .97 & .99 \\
1B & .82 & .94 & .98 & 1.00 \\
2B & .82 & .97 & 1.00 & .99 \\
3A & .86 & .98 & .99 & 1.00 \\
4A & .77 & .92 & .99 & .99 \\
4B & .81 & .97 & 1.00 & 1.00 \\
Mean & .80 & .95 & .99 & .99 \\
\hline
\end{tabular}

Note $: \mathrm{RR}=$ Response Rate.

identical to those performed on the collapsed scores revealed the same reliable differences among DD categories. These patterns of performance clearly show that the pigeons were sensitive to how perceptually discrepant the $\mathrm{S}^{-}$s were from the $\mathrm{S}^{+}$throughout the entirety of discrimination training.

Generalization (and generalization decrement) across compound stimuli. Our stagewise discrimination training procedure used intermediate dimensional values in order to keep the future relevant dimensional values as close to associatively neutral as possible. This procedure was expected to occasion transient disruptions in discrimination performance during the early sessions of each stage.

To assess the immediate effect of adding new dimensional values to the previously learned compound stimuli from earlier stages, we compared the rate of responding to the $\mathrm{S}^{+}$ during the last session of a given stage against the rate of responding to the new compounds during the first session of the subsequent stage. In order to do so, we first grouped the $\mathrm{S}^{-} \mathrm{s}$ based on their similarity to the $\mathrm{S}^{+}$from the previous stage, using the formula:

$$
\mathrm{S}_{\mathrm{x}}=\frac{\mathrm{e}-\mathrm{i}}{\mathrm{n}}
$$

in which $S_{x}$ is the similarity of compound $x, n$ is the number of extreme dimensional values of compound $\mathrm{x}, \mathrm{e}$ is the number of
Table 5

Mean Discrimination Ratios (DRs) in Each Stage for Different Numbers of Dimensional Disparities (DDs).

\begin{tabular}{|c|c|c|c|c|c|}
\hline \multicolumn{6}{|c|}{ Dimensional Disparities (DD) Discrimination Ratio: } \\
\hline \multicolumn{6}{|c|}{$R R$ to $S^{+} /\left(R R\right.$ to $S^{-} s+R R$ to $\left.S^{+}\right)$} \\
\hline \multirow[b]{2}{*}{ Stage } & \multirow{2}{*}{$\begin{array}{l}\text { Pigeon (Sessions } \\
\text { of Training) }\end{array}$} & \multicolumn{4}{|c|}{ DD DR } \\
\hline & & 1 & 2 & 3 & 4 \\
\hline \multirow[t]{7}{*}{1} & $1 \mathrm{~A} \mathrm{(3)}$ & .73 & & & \\
\hline & $3 \mathrm{~A}(1)$ & .95 & & & \\
\hline & $4 \mathrm{~A}(4)$ & .68 & & & \\
\hline & $1 \mathrm{~B}(3)$ & .81 & & & \\
\hline & 2B (2) & .72 & & & \\
\hline & $4 \mathrm{~B}(2)$ & .71 & & & \\
\hline & Mean (2.5) & .77 & & & \\
\hline \multirow[t]{7}{*}{2} & $1 \mathrm{~A}(3)$ & .74 & .81 & & \\
\hline & $3 \mathrm{~A}(4)$ & .81 & 1.00 & & \\
\hline & $4 \mathrm{~A}(4)$ & .77 & .94 & & \\
\hline & 1B (3) & .78 & .88 & & \\
\hline & $2 \mathrm{~B}(2)$ & .84 & 1.00 & & \\
\hline & $4 \mathrm{~B}(2)$ & .79 & .99 & & \\
\hline & Mean (3.0) & .79 & .94 & & \\
\hline \multirow[t]{7}{*}{3} & 1A (9) & .74 & .91 & .96 & \\
\hline & $3 \mathrm{~A}(3)$ & .88 & 1.00 & 1.00 & \\
\hline & $4 \mathrm{~A}(11)$ & .74 & .89 & .98 & \\
\hline & $1 \mathrm{~B}(8)$ & .80 & .95 & .97 & \\
\hline & 2B (6) & .81 & .99 & 1.00 & \\
\hline & $4 \mathrm{~B}(14)$ & .78 & .94 & 1.00 & \\
\hline & Mean (8.5) & .79 & .95 & .99 & \\
\hline \multirow[t]{8}{*}{4} & $1 \mathrm{~A}(19)$ & .80 & .97 & .98 & .99 \\
\hline & $3 \mathrm{~A}(8)$ & .87 & .95 & .99 & 1.00 \\
\hline & $4 \mathrm{~A}(32)$ & .79 & .93 & .99 & .99 \\
\hline & $1 \mathrm{~B}(10)$ & .85 & .96 & .99 & 1.00 \\
\hline & 2B (56) & .81 & .97 & 1.00 & .99 \\
\hline & $4 \mathrm{~B}(17)$ & .85 & .99 & 1.00 & 1.00 \\
\hline & Mean (23.7) & .83 & .96 & .99 & .99 \\
\hline & Overall Mean (37.7) & .80 & .95 & .99 & .99 \\
\hline
\end{tabular}

compound x's extreme dimensional values involved in the $\mathrm{S}^{+}$from the previous stage, and $\mathrm{i}$ is the number of compound $\mathrm{x}$ 's nonnovel and extreme dimensional values never involved in the $\mathrm{S}^{+}$from the previous stage. Positive indexes denote similarity to the $\mathrm{S}^{+}$ from the previous stage, whereas negative indexes denote similarity to the pure $\mathrm{S}^{-}$ (a stimulus created from dimensional values that never were reinforced during discrimination training). Table 6 shows the similarity indexes for the different compound stimuli that a sample pigeon (1B) received across stages.

Three features of this calculation deserve attention. First, the largest positive similarity index in each stage is assigned to the two compound stimuli that derived from the $\mathrm{S}^{+}$from the previous stage, whereas the remaining 
similarity indexes correspond to compound stimuli that derived from the $\mathrm{S}^{-} \mathrm{s}$ from the previous stage. Following the example shown in Table 6, in Stage 2, both CL and CS derive from the $\mathrm{S}^{+}$from Stage $1(\mathrm{C})$, whereas both SL and SS derive from the prior $\mathrm{S}^{-}(\mathrm{S})$. Second, positive indexes denote stimuli composed mainly by dimensional values involved in the previous $\mathrm{S}^{+}$, whereas negative indexes denote stimuli composed mainly by dimensional values never involved in the previous $\mathrm{S}^{+}$. Note in Table 6 that compound CLVL shares two out of its three non-novel dimensional values with the $\mathrm{S}^{+}$from the previous stage $(\mathrm{CLH})$, whereas compound CSVL shares only one value. Third, the formula produces different levels of similarity across stages, capturing not only the degree of similarity between compounds, but also the degree of generalization decrement that might be produced by the introduction of new dimensional values; this is because the ratio of novel to familiar dimensional values falls from 1:1 in Stage 2, to 1:2 in Stage 3, and to 1:3 in Stage 4. Note in Table 6 that even though both CS and CLHL compounds derive from the $\mathrm{S}^{+}$of a previous stage (C and CLH, respectively), the similarity index of the former is smaller than that of the latter. This is due to the number of extreme (non-intermediate) dimensional values involved in the compounds.

After categorizing the different compounds by their similarity to the $\mathrm{S}^{+}$from the previous stage, we expressed the response rate to each of the compounds during the first session of each stage as a percentage of the response rate to the $\mathrm{S}^{+}$from the previous stage, during the last session of that stage (\%RR). Of greatest importance to this analysis, robust generalization of responding from the dimensional values of the $\mathrm{S}^{+}$from the previous stage and/or generalization decrement from the $\mathrm{S}^{-} \mathrm{s}$ from the previous stage should result in elevated responding to the new $\mathrm{S}^{-}$s, thereby producing \%RRs greater than $20 \%$ (our original discrimination learning criterion). Additionally, generalization decrement from the $\mathrm{S}^{+}$ from the previous stage should result in decreased responding to the new $\mathrm{S}^{+}$, thereby producing \%RRs smaller than $100 \%$ (the response rate to the $\mathrm{S}^{+}$from the previous stage). Finally, we expected the $\%$ RR scores to be proportional to the degree of similarity shared between a given $\mathrm{S}^{-}$and the $\mathrm{S}^{+}$from the previous stage.

Figure 3 depicts the $\% \mathrm{RR}$ scores as a function of the compound similarity index in the final three training stages. First, note that separate white bars depict the $\mathrm{S}^{+} \mathrm{s}$ in each stage. Each $\mathrm{S}^{+}$has the same similarity index as the $\mathrm{S}^{-}$ represented by the bar immediately to its left (light-gray bars), because they each contain all of the dimensional values that composed the $\mathrm{S}^{+}$from the previous stage. For example, during Stage 2 for Pigeon 1B (Table 6), both $\mathrm{CL}$ and CS derive from the $\mathrm{S}^{+}$from Stage $1(\mathrm{C})$ and thus share the same similarity index $(+.50)$; however, CL was the current $\mathrm{S}^{+}$, whereas CS was one of the $\mathrm{S}^{-}$s.

It is evident that the \%RRs of these two compounds were very similar in every stage; these pairs of stimuli supported high rates of responding, even though one member of each compound was now the $\mathrm{S}^{+}$and the other member was an $\mathrm{S}^{-}$. Clearly, the newly added dimensional values had not yet gained discriminative control of responding, nor did these newly added dimensional values consistently lower the pigeons' rate of responding.

Table 6

Similarity Indexes of the Compound Stimuli of Pigeon 1B.

\begin{tabular}{|c|c|c|c|c|c|c|}
\hline \multicolumn{7}{|c|}{ Similarity Index: $(\mathrm{e}-\mathrm{i}) / \mathrm{n}$} \\
\hline Stage & $\mathrm{S}^{+}$from previous stage & Compounds & $\mathrm{n}$ & $\mathrm{e}$ & $\mathrm{i}$ & Similarity Index \\
\hline \multirow[t]{2}{*}{2} & $\mathrm{C}$ & CL, CS & 2 & 1 & 0 & +.50 \\
\hline & & SL, SS & & 0 & 1 & -.50 \\
\hline \multirow[t]{3}{*}{3} & CL & CLH, CLV & 3 & 2 & 0 & +.67 \\
\hline & & CSH, CSV, SLH, SLV & & 1 & 1 & 0.00 \\
\hline & & $\mathrm{SSH}, \mathrm{SSV}$ & & 0 & 2 & -.67 \\
\hline \multirow[t]{4}{*}{4} & CLH & CLHD, CLHL & 4 & 3 & 0 & +.75 \\
\hline & & CLVL, CLVD, CSHL, CSHD, SLHL, SLHD & & 2 & 1 & +.25 \\
\hline & & CSVL, CSVD, SSHL, SSHD, SLVL, SLVD & & 1 & 2 & -.25 \\
\hline & & SSVD, SSVL & & 0 & 3 & -.75 \\
\hline
\end{tabular}




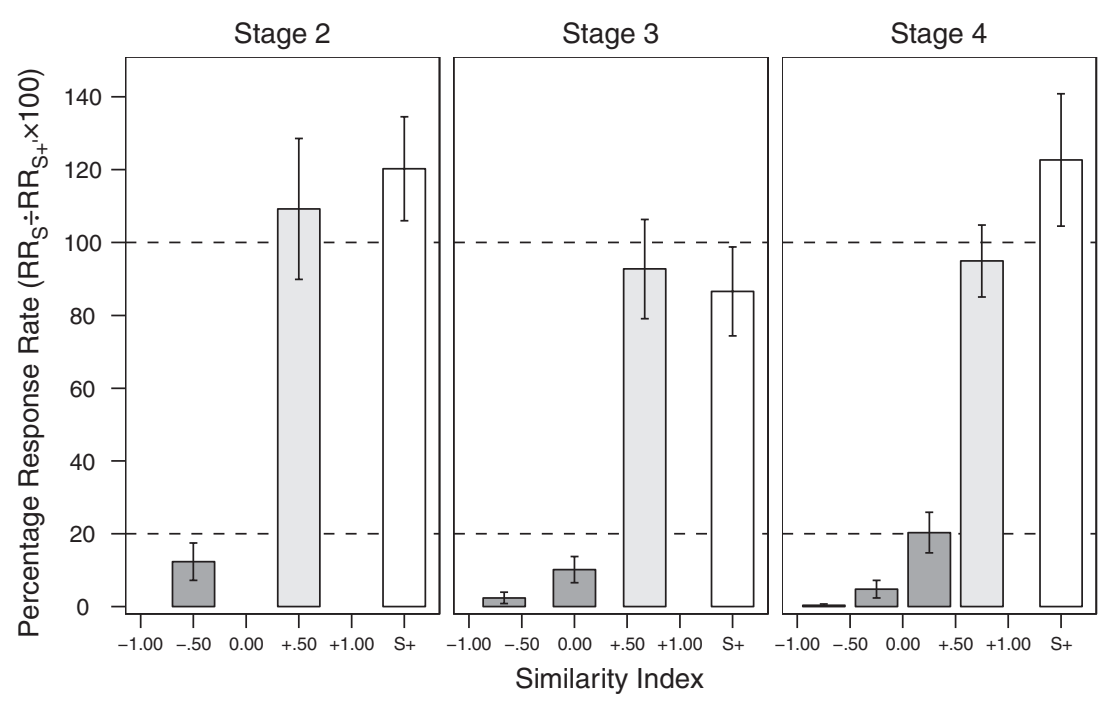

Fig. 3. Mean response rate (RR) to novel compounds during the first session of each stage, expressed as a percentage of the RR to the $S^{+}$during the last session of the previous stage. $R_{S}=$ mean $R R$ to the compounds with the same similarity index; $\mathrm{RR}_{\mathrm{S}^{+}}{ }^{\prime}=\mathrm{RR}$ to the $\mathrm{S}^{+}$during the last session of the previous stage. Positive similarity indexes denote compounds composed mainly by dimensional values included in the $\mathrm{S}^{+}$from the previous stage, whereas negative similarity indexes denote compounds composed mainly by dimensional values not included in the $\mathrm{S}^{+}$from the previous stage. The $\mathrm{S}^{+}$compound in each stage is plotted separately (white bar in each panel), but its similarity index is equal to the largest positive similarity index possible in each stage (light gray bar in each panel). Both the $\mathrm{S}^{+}$compounds and the compounds with the largest positive similarity index possible in each stage derive from the $\mathrm{S}^{+}$compound in the previous stage, whereas the remaining compounds (dark gray bars) derive from the $\mathrm{S}^{-}$compounds in the previous stage. Refer to the main text for the formula used to calculate the similarity index. The lower horizontal dashed line denotes the percentage value expected for $\mathrm{S}^{-} \mathrm{s}$ at criterion performance, whereas the upper horizontal dashed line denotes the percentage at which the $R R$ to the novel compounds is equal to the $R R$ to the $S^{+}$from the previous stage.

Furthermore, the introduction of novel dimensional values to the remaining $\mathrm{S}^{-} \mathrm{s}$ (darkgray bars) did not result in \% RR scores that surpassed the $20 \%$ response rate criterion; the introduction of novel dimensional values did not result in the loss of discriminative control by the $\mathrm{S}^{-} \mathrm{s}$ from the previous stage. Finally, within that group of $\mathrm{S}^{-} \mathrm{s}, \% \mathrm{RR}$ scores were proportional to the degree of similarity that the $\mathrm{S}^{-} \mathrm{s}$ had with the $\mathrm{S}^{+}$from the previous stage; the individual dimensional values did exert some control over discriminative behavior albeit over a very small range of responding.

One-sample, one-tailed $t$-tests revealed that, in every stage, the \%RRs to the $\mathrm{S}^{-}$ compound stimuli that derived from the previous $\mathrm{S}^{+}$(light-gray and white bars in Fig. 3) were reliably larger than $20 \%$ (all $p \mathrm{~s}<.01)$, and were not reliably smaller than $100 \%$ (all $p$ s $>.10$ ). Furthermore, none of the \%RRs to compound stimuli that derived from previous $\mathrm{S}^{-} \mathrm{s}$ (dark-gray bars in Fig. 3) reliably surpassed the $20 \%$ criterion (all $p$ s > .10). To complete the assessment, we compared the \%RRs among similarity levels using multiple pairwise comparisons with a Bonferroni correction. In Stage 2, the \%RR score to the -.50 compounds was reliably lower than the scores to the +.50 and $\mathrm{S}^{+}$ compounds (both $p \mathrm{~s}<.001$ ), but the $\% \mathrm{RR}$ scores to +.50 and $\mathrm{S}^{+}$compounds were not reliably different from one another $(p>.10)$. In Stage 3, the \%RR scores between the -.67 and 0 compounds, and between the +.67 and $\mathrm{S}^{+}$compounds were not reliably different (both $p \mathrm{~s}>.10$ ), but all other possible comparisons between the compounds were reliably different (all $p$ s $<$ $.001)$. Finally, the Stage 4 results confirmed the same trend: Differences among the -.75, -.25 , and +.25 compounds, and between the +.75 and $\mathrm{S}^{+}$compounds were not reliable (all $p \mathrm{~s}>.10$ ), whereas all of the differences between members of these two subgroups were reliable (all $p \mathrm{~s}<.001$ ).

These results confirm that the introduction of novel dimensional values did not result in the resurgence of responding to the $\mathrm{S}^{-}$ 

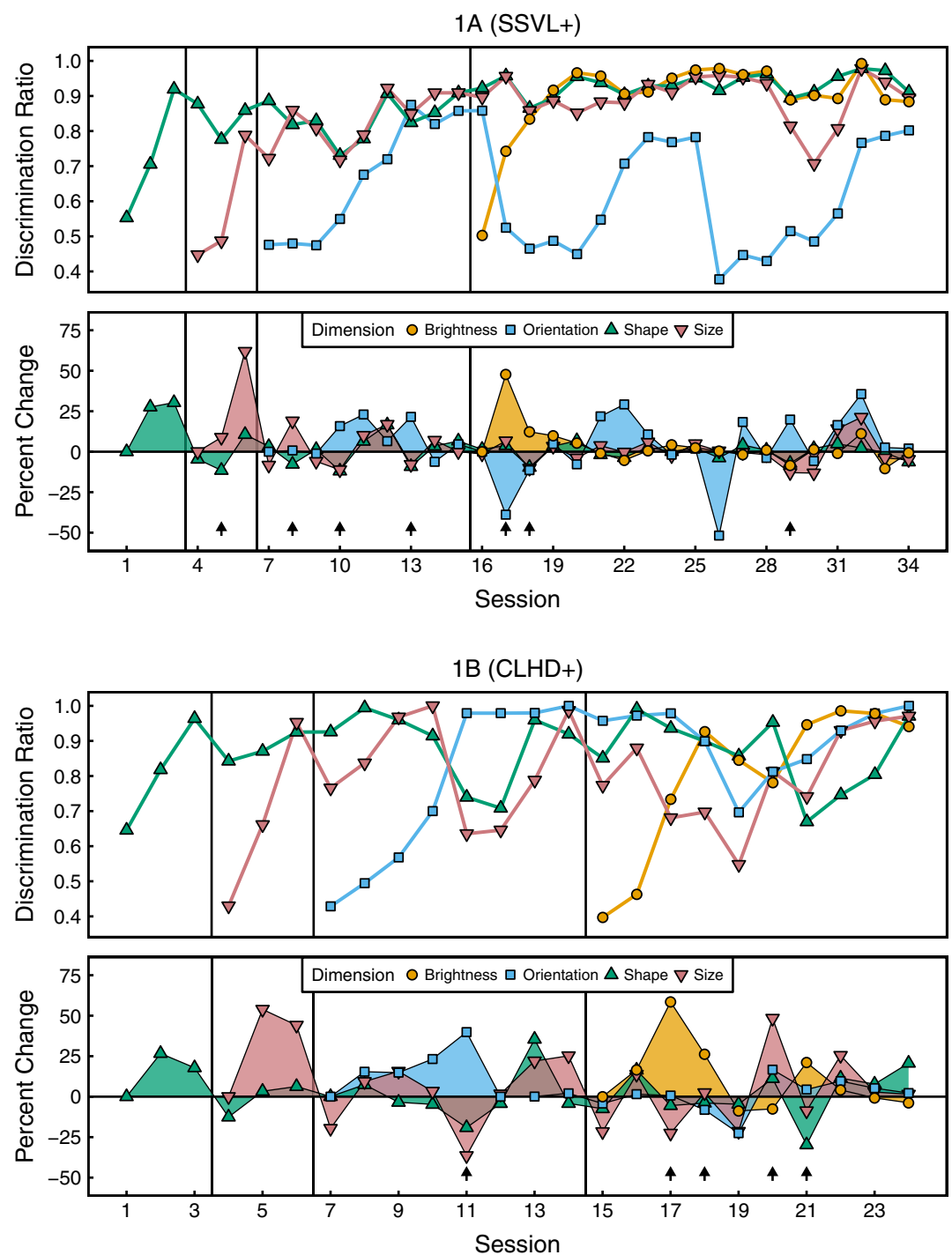

Fig. 4. Dimensional discrimination ratios (DRs) for shape, size, orientation, and brightness throughout the entirety of discrimination training (upper portion), and percent change in DR from the previous session (lower portion) for each pigeon. The vertical lines mark the addition of a new discriminative dimension. The arrows in the bottom panel denote attentional tradeoffs between dimensions. See the online version of this article for a colored version of this figure.

compounds or in the reduction of responding to the $\mathrm{S}^{+}$compounds from the previous stage. No evidence suggesting the presence of generalization decrement was found.

Attentional tradeoffs: individual pigeons' dimensional discrimination ratios. We next considered the relation between the dimensional DRs observed throughout the course of discrimination training to assess the dynamics of any changes in responding on the stagewise MNC task. The upper portion of each graph in
Figure 4 shows the dimensional DRs of each pigeon across training sessions. The lower portion of each graph in Figure 4 shows the percentage change in the dimensional DRs of each pigeon using the previous session as a reference point; an upward change indicates an improvement of the discrimination of a dimension, whereas a downward change indicates a worsening of the discrimination of a dimension. If attentional tradeoffs were to occur between dimensions, then an upward change 

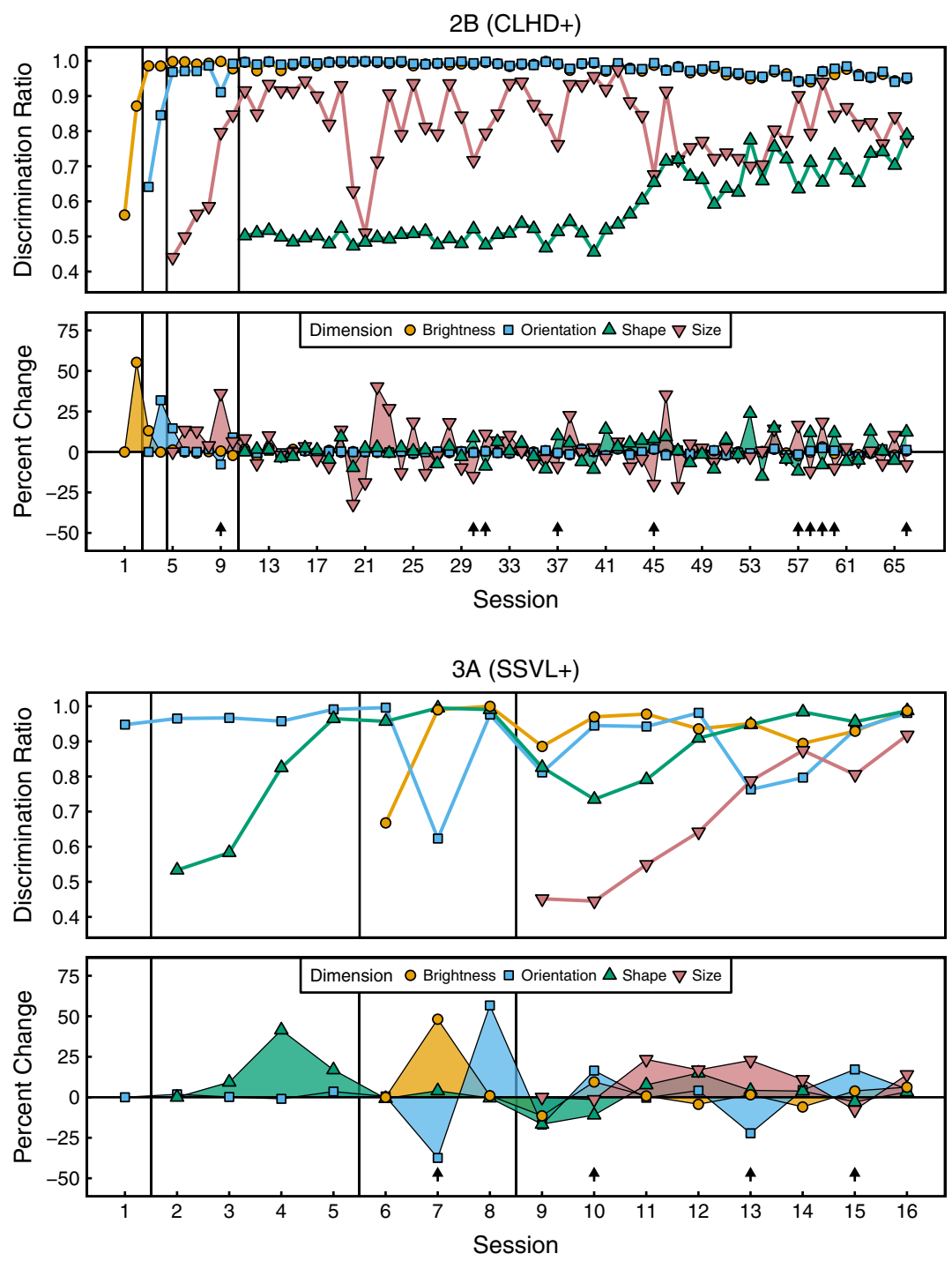

Fig. 4. Continued

in discrimination performance to one dimension should be accompanied by a downward change in discrimination performance to another dimension.

We identified attentional tradeoffs using a joint criterion. An attentional tradeoff involved both upward and downward change in two of more dimensions of at least $7.5 \%$ (which yields at least a $15 \%$ separation between dimensions). Upward-pointing arrows in the bottom panel of Figure 4 denote these attentional tradeoffs.

All pigeons showed signs of attentional tradeoffs in Stages 3 and 4; signs of attentional tradeoffs were less evident in Stage 2; and, they could not occur in Stage 1, when the pigeons were required to discriminate values along only one dimension. It is important to note that most of these tradeoffs involve just two dimensions, which supports the idea that attentional tradeoffs are not an artifact of factors that may generally affect discriminative responding. For example, if a pigeon were to be more motivated to peck for food in a given session, then all of the dimensional DRs for the stimuli presented should be similarly affected.

The training sequence for Pigeon 1A was shape-size-orientation-brightness. Adding the 

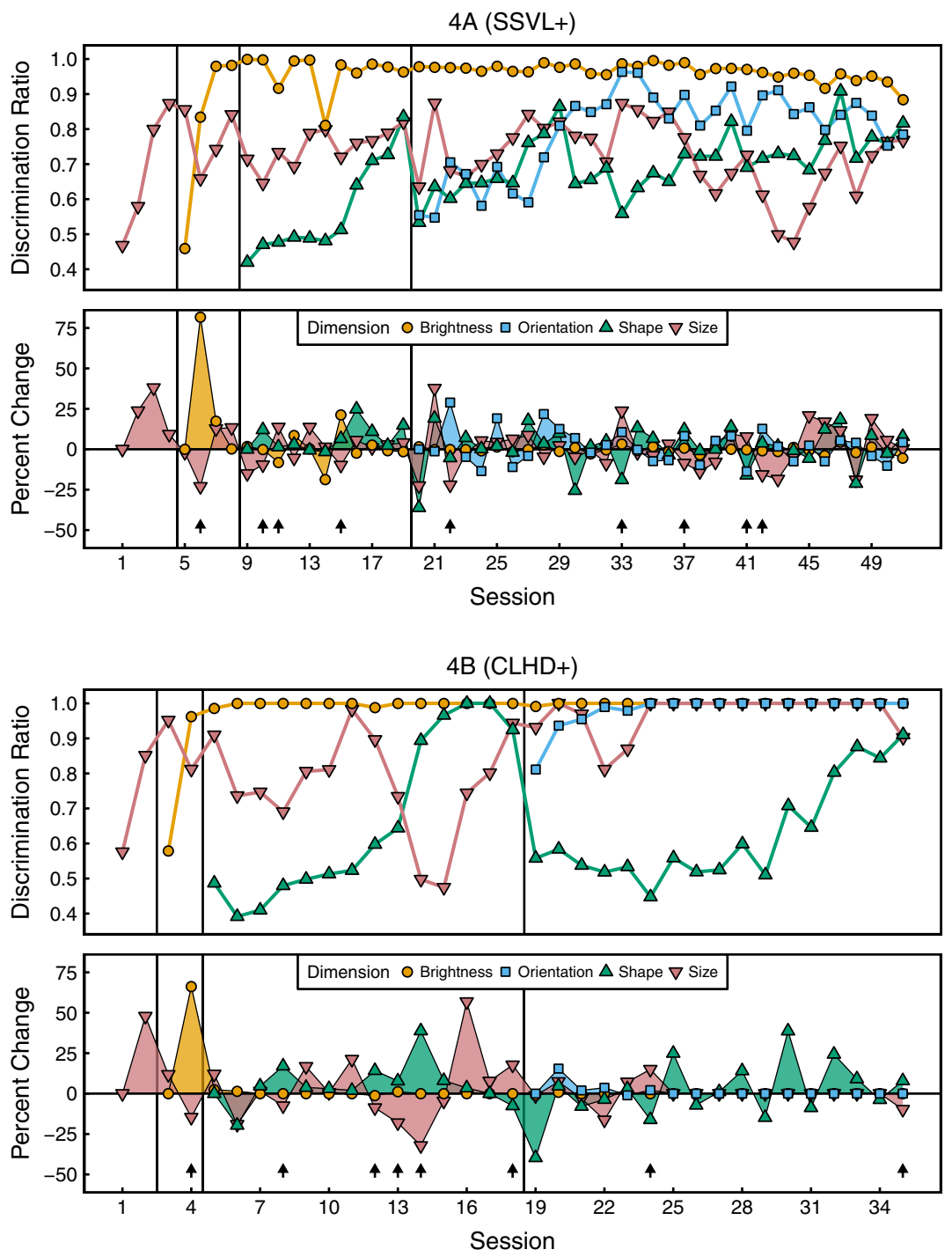

Fig. 4. Continued

size dimension in Stage 2 resulted in a very slight and brief decrease in the shape DR as the size DR inched upward in Session 5. When orientation was added in Stage 3, a brief tradeoff between the already acquired shape and size discriminations occurred in Session 8, and the DRs for both of these dimensions fell as the orientation DR rose in Session 10. When brightness was added in Stage 4, the orientation DR dramatically dropped as the pigeon began to acquire discriminative performance to brightness in Sessions 17 and 18; a similar, but less dramatic pattern was repeated in Session 29, but now between the brightness and size DRs.
Pigeon 1B was trained with the same training sequence as Pigeon 1A. When the extreme values for size were introduced in Stage 2, there were no noticeable tradeoffs. Striking tradeoffs did occur in Session 11 in Stage 3 , where an increase in discrimination of the newly introduced orientation dimension produced a sharp decline in discrimination for both the size and shape dimensions. Finally, when brightness was introduced in Stage 4 , clear tradeoffs involved brightness and the remaining dimensions. Tradeoffs with size in Sessions 17 and 20, orientation in Session 18, and shape in Session 21 were observed. 
The training sequence for Pigeon 2B was brightness-orientation-size-shape. Stage 2 was extremely short, without any tradeoffs. Stage 3 was also short, with one very small and brief attentional tradeoff occurring in Session 9. Stage 4 proved to be extremely long, with the clearest signs of two instances of repeated attentional tradeoffs starting in Sessions 28 and 57, where rises in the newly introduced shape dimension DR came at the expense of falls in the size dimension DR.

Pigeon 3A was the fastest subject to complete training. Its training sequence was orientation-shape-brightness-size. No tradeoffs were observed in Stage 2. However, adding the brightness dimension in Stage 3 produced a pronounced decrease in the DR of the orientation dimension in Session 7 when the DR of the brightness dimension rose abruptly. Finally, in Stage 4, two tradeoffs between orientation and the newly added size dimension occurred in Sessions 13 and 15.

Pigeon 4A experienced the training sequence size-brightness-shape-orientation. Although it was brief, Stage 2 involved a striking size/brightness tradeoff in Session 6. Stage 3 showed a small tradeoff between newly relevant shape dimension and size in Session 10 , which was followed by a tradeoff between size and brightness in Sessions 11 and 15. Additionally, Stage 4-which was quite longappeared to involve many tradeoffs. Attentional tradeoffs between the DR of the newly added orientation dimension and the size dimension occurred in Sessions 22, 41, and 42. Other tradeoffs occurred between shape and size in Sessions 33 and 37. Interestingly, brightness did not participate in any attentional tradeoffs during this stage, as its DR remained high throughout.

Pigeon $4 \mathrm{~B}$ shared the training sequence of Pigeon 4A. Although Stage 2 was brief, there was a tradeoff between size and the newly added brightness dimension in Session 4. Stage 3 involved a prolonged and pronounced tradeoff (spanning Sessions 12, 13, 14, and 18) between size and the newly added dimension of shape. The addition of the orientation dimension in Stage 4 produced an immediate, dramatic, and long-lived decrease in the shape DR, although with no corresponding increases in discrimination of any of the other dimensions. Throughout Stages 3 and 4, the brightness DR remained at high levels; however, small tradeoffs between shape and size were seen in Sessions 24 and 35.

\section{Discussion}

Deploying a stagewise version of the $\mathrm{MNC}$ discrimination task, we again observed the occurrence of attentional tradeoffs between discriminative behavior controlled by previously learned and newly emerging discriminative stimuli (Fig. 4). Using this method, we also replicated the main observations made in our two most recent MNC discrimination projects (Vyazovska et al., 2014; Teng et al., 2015): (1) discrimination performance was positively related to the number of dimensional disparities between the $\mathrm{S}^{+}$and $\mathrm{S}^{-}$compound discriminative stimuli (Fig. 2); (2) overall, there were small, unreliable differences among the salience of the four discriminative dimensions, although there were reliable differences among the individual pigeons (Table 2); and (3) the duration of training required to achieve discrimination criterion increased as the number of relevant discriminative dimensions was increased from 1 to 4 (Fig. 4).

Both the original MNC discrimination task and its stagewise variant arrange the same end-point discrimination; however, it is likely that the pigeons may have to learn the tasks differently. When all of the dimensional values are present from the outset of training, the pigeons' main challenge is to discriminate among some 16 compound stimuli. In contrast, when the MNC discrimination is progressively trained across stages, pigeons may have to layer more complex discriminations on top of simpler learned discriminations (Fig. 1).

Consider the transition from Stage 1 to Stage 2. In Stage 1, our pigeons discriminated stimuli that differed along a single dimensional value $\left(\mathrm{Al}^{+}\right.$vs. $\left.\mathrm{A2}^{-}\right)$. However, in Stage 2 -with the addition of another pair of dimensional values - that simple discrimination was no longer sufficient; our pigeons now had to discriminate that two new compound-stimulus versions of the previously positive stimulus $\left(\mathrm{A} 1^{+}\right)$were differentially associated with the delivery of food $\left(\mathrm{A} 1 \mathrm{~B} 1^{+}\right.$vs. A1B2- $)$. Our data suggest that after a new pair of dimensional values was added in each stage, our pigeons kept responding at virtually the same rate to the novel $\mathrm{S}^{-}$compounds containing the specific dimensional value(s) that had been 
reinforced in the previous stage (Fig. 3, lightgray bars). That high rate of responding subsequently fell due to nonreinforcement.

Therefore, in each stage, an improvement in overall discriminative performance had primarily to establish a discrimination between the $\mathrm{S}^{+}$and $\mathrm{S}^{-}$compound stimuli that derived from the $\mathrm{S}^{+}$from the previous stage (see Fig. S1 in the online supplemental materials for similarity-based DRs throughout the entirety of training). The new S- compounds that derived from the $\mathrm{S}^{-}$from the previous stage immediately and appropriately controlled low rates of responding (Fig. 3, darkgray bars).

A key objective of our stagewise MNC discrimination task was to minimize the involvement of learned irrelevance, which could have contributed to an earlier study investigating stagewise discrimination training (Gottselig et al., 2001). In that prior study, both of the newly relevant dimensional values had previously been nondifferentially reinforced; in the present study, we dealt with that complication by scheduling intermediate dimensional values. Although this tactic does not prevent the development of learned irrelevance to each dimension as a whole, it does prevent the development of learned irrelevance to the specific dimensional values that were to be used in subsequent stages of discrimination training. Given that performance in our task was similar to performance in Gottselig et al.'s (2001) task, we conclude that learned irrelevance probably did not play an important role in their results.

Nevertheless, our stagewise procedure introduces an additional complication. Adding completely novel dimensional values could have produced generalization decrement (Pearce, 1987), thus attenuating the control exerted by previously learned compound stimuli. Our results indicated that was not the case. Introducing novel dimensional values did not result in increased responding to previously nonreinforced compounds (Fig. 3, dark-gray bars) nor did it result in decreased responding to previously reinforced compounds (Fig. 3, light-gray and white bars).

However, even if our methods and analyses were not sensitive enough to detect it, the early disruption in the control prompted by the introduction of novel compound stimuli should not prevent the occurrence of attentional shifts; yet, attentional shifts were in fact seen after the new dimensional values were introduced. From the perspective of selective attention (Kruschke \& Johansen, 1999; Pashler, 1998; Riley \& Roitblat, 1978; Sutherland \& Mackintosh, 1971; Thomas, 1970; Trabasso \& Bower, 1968), the tradeoffs we observed between the dimensional DRs are the likely result of the pigeon's limited attentional capacity.

To conclude, the observation of attentional shifts in the pigeon given the MNC discrimination task has now been obtained in a multiplicity of settings. These demonstrations both document the robustness of the phenomenon and underscore the utility of the MNC discrimination task. We believe that the MNC task-in any of its variants-represents a valuable methodology with which to study the roles of selective attention and stimulus control involving the discrimination of complex conditioned stimuli.

\section{References}

Castro, L., \& Wasserman, E. A. (2013). Informationseeking behavior: Exploring metacognitive control in pigeons. Animal Cognition, 16, 241-254. doi: 10.1007/ s10071-012-0569-8

Chatlosh, D. L., \& Wasserman, E. A. (1993). Multidimensional stimulus control in pigeons: Selective attention and other issues. In T.R. Zentall (Ed.), Animal cognition: A tribute to Donald A. Riley (pp. 271-292). Hillsdale, NJ: Erlbaum.

Gottselig, J. M., Wasserman, E. A., \& Young, M. E. (2001). Attentional tradeoffs in pigeons learning to discriminate newly-relevant visual stimulus dimensions. Learning and Motivation, 32, 240-253. doi: 10.1006/ lmot.2000.1081

Kruschke, J. K., \& Johansen, M. K. (1999). A model of probabilistic category learning. Journal of Experimental Psychology: Learning, Memory, and Cognition, 25, 1083-1119. doi: 10.1037/0278-7393.25.5.1083

Mackintosh, N. J. (1973). Stimulus selection: Learning to ignore stimuli that predict no change in reinforcement. In R. A. Hinde \& J. Stevenson-Hinde (Eds.), Constraints on learning. London: Academic Press.

Mackintosh, N. J. (1975). A theory of attention: Variations in the associability of stimuli with reinforcement. Psychological Review, 82, 276-298. doi: 10.1037/h0076778

Pashler, H. E. (1998). The psychology of attention. Cambridge, MA: MIT Press.

Pearce, J. M. (1987). A model for stimulus generalization for Pavlovian conditioning. Psychological Review, 94, 61-73. 10.1037/0033-295X.94.1.61

Riley, D. A., \& Roitblat, H. L. (1978). Selective attention and related cognitive processes in pigeons. In S. H. Hulse, H. Fowler, \& W. K. Honig (Eds.), Cognitive processes in animal behavior (pp. 249-276). Hillsdale, NJ: Erlbaum.

Soto, F. A., \& Wasserman, E. A. (2010). Integrality/separability of stimulus dimensions and multidimensional 
generalization in pigeons. Journal of Experimental Psychology: Animal Behavior Processes, 36, 194-205. $10.1037 / \mathrm{a} 0016560$

Sutherland, N. S., \& Mackintosh, N. J. (1971). Mechanisms of animal discrimination learning. New York: Academic Press.

Thomas, D. R. (1970). Stimulus selection, attention, and related matters. In J. H. Reynierse (Ed.), Current issues in animal learning (pp. 311-356). Lincoln, NE: University of Nebraska Press.

Trabasso, T., \& Bower, G. H. (1968). Attention in learning. New York: Wiley.

Treisman, A. (1964). Selective attention in man. British Medical Bulletin, 20, 12-16. http://bmb. oxfordjournals.org/content/20/1/12.citation
Teng, Y., Vyazovska, O. V., \& Wasserman E. A. (2015). Selective attention and pigeons' multiple necessary cues discrimination learning. Behavioural Processes, 112, 61-71. 10.1016/j.beproc.2014.08.004

Vyazovska, O. V., Teng, Y., \& Wasserman E. A. (2014). Attentional tradeoffs in the pigeon. Journal of the Experimental Analysis of Behavior, 101, 337-354. 10.1002/ jeab. 82

Zentall, T. R. (2012). Selective and divided attention in birds. In O.F. Lazareva, T. Shimizu, \& E. A. Wasserman (Eds.), How animals see the world (pp. 351-369). New York: Oxford University Press.

Received: March 31, 2016 Final Acceptance: May 31, 2016 